\title{
Grundwasserschutz in urbanen Räumen der Schweiz
}

\section{Adrian Auckenthaler ${ }^{1}$}

Eingegangen: 1. März 2019 / Online publiziert: 5. April 2019

๑) Springer-Verlag GmbH Deutschland, ein Teil von Springer Nature 2019

Viele von uns leben in dicht besiedelten urbanen Gebieten. Für die Schweiz betrachtet, liegen die Siedlungs- und Gewerbegebiete meist in den größeren Tälern und dem Mittelland, wo auch die bedeutenden Lockergesteinsgrundwasservorkommen sind. Dieses Grundwasser versorgt rund $80 \%$ der Bevölkerung mit Trinkwasser. Für die Fassungen wurden in den 1970er und 1980er Jahren Schutzzonen (S1, S2 und S3) ausgeschieden. Die S2 soll möglichst frei von Nutzungen sein und dient dem Schutz vor mikrobiellen Verunreinigungen. Von deren Grenze soll das Grundwasser mindestens 10 Tage im Untergrund fließen, bis es zur Fassung gelangt.

Leider wurden die Fließzeiten des Grundwassers, die man für die Dimensionierung der Schutzzonen herangezogen hatte, in etlichen Fällen deutlich unterschätzt. Dies führt dazu, dass die überarbeiteten, nun hydrogeologisch korrekten Schutzzonen deutlich größer werden. In der Zwischenzeit sind jedoch auch die Siedlungsgebiete gewachsen, sodass Nutzungskonflikte vorprogrammiert sind.

Das schweizerische Bundesamt für Umwelt (BAFU) hat bei einer Umfrage in den Kantonen festgestellt, dass rund 1 Mio. Menschen in der Schweiz Trinkwasser von ungenügend geschützten Fassungen trinken. Alternative Standorte für diese Fassungen sind kaum zu finden, da ein Großteil der Flächen über den Grundwasserleitern bereits besiedelt ist. Wegen der Versorgungssicherheit müssen die Fassungen meist trotzdem weiter betrieben werden. Es gilt also, die noch zu schützenden Flächen für den Grundwasserschutz möglichst freizuhalten.

Was sind nun aber die Auswirkungen des mangelhaften Grundwasserschutzes auf die Grundwasserqualität? Ist es für die Trinkwasserkonsumenten wirklich ein Problem,

Adrian Auckenthaler

adrian.auckenthaler@bl.ch

1 Amt für Umweltschutz und Energie, Kanton Basel-Landschaft, Bau- und Umweltschutzdirektion, Rheinstr. 29, 4410 Liestal, Schweiz wenn die Schutzzonen zu klein dimensioniert sind? Und was ist die richtige Bemessung für eine Schutzzone? Im Vergleich zu Deutschland und Österreich sind die Schutzzonen S2 in der Schweiz sehr klein bemessen.

In seltenen Fällen führen die zu kleinen Schutzzonen direkt zu fäkalen Verunreinigungen des Trinkwassers. Pumpwerke mit zeitweise größeren Verunreinigungen liegen praktisch ausschließlich flussnah mit einem bedeutenden Anteil an jungem Flusswasserinfiltrat. Jedoch findet man fast in jedem für Trinkwasser genutztem Grundwasser Mikroverunreinigungen. Gerade Haushaltchemikalien stammen hingegen nicht nur aus via Flüssen infiltriertem gereinigten Abwasser. Neuere Untersuchungen in der Nordwestschweiz zeigen, dass Medikamente, Lebensmittelzusatzstoffe und weitere Chemikalien auch über lecke Kanalisationen ins Grundwasser eingetragen werden und wesentlich zur allgemeinen Belastung beitragen können.

Die zu kleinen Schutzzonen bewirken also nicht per se eine Belastung des Grundwassers. Sie bilden aber einen kleineren natürlichen Filter und führen dadurch zu einer höheren Gefährdung. Diese manifestiert sich bis heute jedoch kaum. Bei Nutzungskonflikten durch die Erweiterung von Schutzzonen in urbanen Räumen sollen die Fassungen deshalb, sofern die Wasserqualität nicht negativ beeinflusst ist, nicht aufgegeben werden. Es sollen jedoch griffige technische Maßnahmen getroffen werden, um das Versickern von verschmutztem Abwasser sicher zu verhindern. Diese Aufgabe müssen die Wasserversorgungen veranlassen und falls die Nutzungseinschränkungen für die Betreiber einer Anlage zu groß sind, auch finanzieren.

Die lokal begrenzen Maßnahmen in den Grundwasserschutzzonen reichen jedoch nicht aus. Für die Reduktion von Mikroverunreinigungen im Grundwasser braucht es einen wirkungsvollen Gewässerschutz. Dabei spielt die Zusammenarbeit der Akteure in verschiedenen Sektoren wie der Wasserwirtschaft, der Raumplanung und der Politik und von uns allen als Trinkwasserkonsumenten eine entscheidende Rolle. 